\title{
General relativistic solitons
}

\author{
A. C. Cadavid \\ Department of Physics and Astronomy, California State University, Northridge, California 91330 \\ R. J. Finkelstein \\ Department of Physics and Astronomy, University of California, Los Angeles, California 90095-1547
}

(Received 31 October 1997; published 29 May 1998)

\begin{abstract}
A general relativistic and nontopological soliton is constructed by coupling a nonlinear scalar field to the standard gravitational field. Our results replicate the basic features of the special relativistic case: namely, a singularity-free lump with a discrete spectrum of eigensolutions. The central singularities and horizon that appear in the black hole solitons of string theory are not present. [S0556-2821(98)05912-8]
\end{abstract}

PACS number(s): 04.62.+v, 04.25.-g, 11.10.Lm

\section{INTRODUCTION}

Since the discovery of solitons in string theory by Strominger [1] in 1990, many other multidimensional solitons have been discussed. Some of these bear a greater resemblance to black holes, however, then to classical solitons and have been called "solitonic black holes" since they carry Schwarzchild-like singularities. They are supposed to be physically allowed if the singularity lies inside the event horizon according to the hypothesis of cosmic censorship. They are also considered possible candidates for the role of preons.

This picture of an elementary particle differs from the earlier conception of an elementary particle demanded by a unitary field theory, where the particles have no independent existence but are simply singularity-free lumps of field. A well known early attempt at such a theory (Born and Infeld) [2] did succeed in the construction of solitonic lumps but these field structures still carried central singularities. Consequently the point particles were not purged from the theory but persisted as singularities.

Since that time singularity-free solitons have been constructed at the special relativistic level [3-5]. We are here interested in exploring this question at the general relativistic level. It is a very familiar fact that macroscopic balls of fluid, such as stars, may be held in equilibrium by the compensation of hydrodynamic forces by gravitational forces; and in these examples there is no singularity at the origin. In the standard treatment of a star the gravitational field is compensated by pressure and density fields related by an equation of state. Here we should like to consider the possibility that the gravitational field is compensated not by phenomenological fields such as pressure and density but by fundamental fields which do not become singular at the origin and which do not force the gravitational field to become singular either.

If $S$ is the total action, we need to investigate the simultaneous equations

$$
\begin{gathered}
\frac{\delta S}{\delta g^{\mu \lambda}}=0, \\
\frac{\delta S}{\delta \psi^{k}}=0 .
\end{gathered}
$$

Here Eq. (1.1) abbreviates the gravitational equations: namely,

$$
R_{\mu \lambda}=K \Theta_{\mu \lambda}, \quad K=-\frac{8 \pi k}{c^{2}}
$$

and Eq. (1.2) is intended to represent the equations of motion of all the matter fields contributing to $\Theta_{\mu \lambda}$, the source of the gravitational field. The solutions of Eqs. (1.1) and (1.2) must satisfy the following boundary conditions in order to represent solitons: all fields must vanish at large radii and all fields must remain everywhere finite including the origin where we shall also require vanishing first derivatives to avoid cusp singularities.

\section{THE NONLINEAR SCALAR FIELD}

Let us consider as the source of the gravitational field a single complex nonlinear scalar field described by the Lagrangian

$$
L=\frac{1}{2} g^{\mu \lambda} \partial_{(\mu} \psi \partial_{\lambda)} \psi^{*}+F\left(|\psi|^{2}\right)
$$

with the following energy-momentum tensor:

$$
\begin{aligned}
\theta_{\mu \lambda} & =\frac{\partial L}{\partial g^{\mu \lambda}}-\frac{1}{2} g_{\mu \lambda} L \\
& =\frac{1}{2} \partial_{(\mu} \psi \partial_{\lambda)} \psi^{*}-\frac{1}{2} g_{\mu \lambda} L .
\end{aligned}
$$

Then

$$
\Theta_{\mu \lambda}=\theta_{\mu \lambda}-\frac{1}{2} g_{\mu \lambda} \theta
$$

where

$$
\theta=g^{\mu \lambda} \theta_{\mu \lambda}
$$




$$
\Theta_{\mu \lambda}=\frac{1}{2} \partial_{(\mu} \psi \partial_{\lambda)} \psi^{*}+\frac{g_{\mu \lambda}}{2} F\left(|\psi|^{2}\right) .
$$

We shall consider only spherically symmetric structures with harmonic time dependence and therefore set

$$
\begin{aligned}
& \partial_{2} \psi=\partial_{3} \psi=0, \\
& \partial_{1} \psi=\frac{\partial \psi}{\partial r}=\psi^{\prime}, \quad \psi=R(r) e^{i \omega t} .
\end{aligned}
$$

Then

$$
\begin{aligned}
& \Theta_{00}=\frac{1 \omega^{2}}{2 c^{2}} R^{2}+\frac{1}{2} F\left(R^{2}\right) g_{00}, \\
& \Theta_{11}=\frac{1}{2}\left(R^{\prime}\right)^{2}+\frac{1}{2} F\left(R^{2}\right) g_{11}, \\
& \Theta_{22}=\frac{1}{2} F\left(R^{2}\right) g_{22}, \\
& \Theta_{33}=\frac{1}{2} F\left(R^{2}\right) g_{33} .
\end{aligned}
$$

\section{GRAVITATIONAL FIELD}

Let us adopt the following metric:

$$
d s^{2}=e^{\nu(r)} d t^{2}-e^{\lambda(r)} d r^{2}-r^{2}\left(d \theta^{2}+\sin ^{2} \theta d \varphi^{2}\right),
$$

where $\nu(r)$ and $\lambda(r)$ are to be determined by the field equations. Then

$$
\begin{gathered}
R_{00}=e^{\nu-\lambda}\left[-\frac{v^{\prime \prime}}{2}+\frac{\nu^{\prime} \lambda^{\prime}}{4}-\frac{\left(\nu^{\prime}\right)^{2}}{4}-\frac{\nu^{\prime}}{r}\right], \\
R_{11}=\frac{\nu^{\prime \prime}}{2}-\frac{\nu^{\prime} \lambda^{\prime}}{4}+\frac{\left(\nu^{\prime}\right)^{2}}{4}-\frac{\lambda^{\prime}}{r}, \\
R_{22}=e^{-\lambda}\left[1+\frac{\nu^{\prime} r}{2}-\frac{\lambda^{\prime} r}{2}\right]-1, \\
R_{33}=R_{22} \sin ^{2} \theta,
\end{gathered}
$$

where the prime denotes differentiation with respect to $r$. Since we are considering the special case in which the nonlinear scalar field is the sole source of the gravitational field, the graviational equations of motion previously denoted by Eq. (1.1), are, by Eqs. (2.8) and (3.2),

$$
\begin{gathered}
{\left[-\frac{1}{2} \nu^{\prime \prime}+\frac{\nu^{\prime} \lambda^{\prime}}{4}-\frac{\left(\nu^{\prime}\right)^{2}}{4}-\frac{\nu^{\prime}}{r}\right]=\frac{K}{2}\left[F e^{\lambda}+\frac{\omega^{2}}{c^{2}} e^{\lambda-\nu} R^{2}\right]} \\
{\left[\frac{1}{2} \nu^{\prime \prime}-\frac{\nu^{\prime} \lambda^{\prime}}{4}+\frac{\left(\nu^{\prime}\right)^{2}}{4}-\frac{\lambda^{\prime}}{r}\right]=\frac{K}{2}\left[-F e^{\lambda}+\left(R^{\prime}\right)^{2}\right]} \\
{\left[1+\nu^{\prime} \frac{r}{2}-\frac{\lambda^{\prime} r}{2}\right]-e^{\lambda}=-\frac{K}{2} F r^{2} e^{\lambda}}
\end{gathered}
$$

By Eqs. (3.3) and (3.4),

$$
-\frac{\nu^{\prime}+\lambda^{\prime}}{r}=\frac{K}{2}\left[\left(R^{\prime}\right)^{2}+\frac{\omega^{2}}{c^{2}} e^{\lambda-\nu} R^{2}\right]
$$

and, by Eqs. (3.5) and (3.6),

$$
-\frac{\lambda^{\prime}}{r}=\frac{e^{\lambda}-1}{r^{2}}-\frac{K}{2} F e^{\lambda}+\frac{K}{4}\left[\left(R^{\prime}\right)^{2}+\frac{\omega^{2}}{c^{2}} e^{\lambda-\nu} R^{2}\right] .
$$

Finally by Eq. (3.6)

$$
\begin{aligned}
& {[\nu+\lambda]_{\infty}-[\nu+\lambda]_{0}} \\
& \quad=-\frac{K}{2} \int d r r\left[\left(R^{\prime}\right)^{2}+\frac{\omega^{2}}{c^{2}} e^{\lambda-\nu} R^{2}\right]>0 .
\end{aligned}
$$

\section{EQUATIONS OF MOTION OF $\psi$}

These equations, denoted by Eq. (1.2) in the Introduction, are

$$
g^{\mu \lambda} \nabla_{\mu} \nabla_{\lambda} \psi-\frac{\partial F\left(|\psi|^{2}\right)}{\partial \psi^{*}}=0,
$$

where

$$
\nabla_{\mu} \psi=\partial_{\mu} \psi
$$

and

$$
\nabla_{\mu} \nabla_{\lambda} \psi=\partial_{\mu} \partial_{\lambda} \psi-\Gamma_{\mu \lambda}^{\sigma} \nabla_{\sigma} \psi
$$

Then

$$
\begin{aligned}
g^{\mu \lambda} \nabla_{\mu} \nabla_{\lambda} \psi & =g^{\mu \lambda} \partial_{\mu} \partial_{\lambda} \psi-g^{\mu \lambda} \Gamma_{\mu \lambda}^{\sigma} \partial_{\sigma} \psi \\
& =g^{00} \partial_{0} \partial_{0} \psi+g^{11} \partial_{1} \partial_{1} \psi-g^{\mu \lambda} \Gamma_{\mu \lambda}^{1} \partial_{1} \psi
\end{aligned}
$$

and Eq. (4.1) becomes by Eqs. (3.1) and (4.3)

$$
R^{\prime \prime}+\frac{1}{2}\left(\nu^{\prime}-\lambda^{\prime}\right) R^{\prime}+\frac{2}{r} R^{\prime}+e^{\lambda-\nu} \frac{\omega^{2}}{c^{2}} R+e^{\lambda} \frac{\partial F}{\partial R}=0 .
$$

\section{PHENOMENOLOGICAL FIELDS}

The soliton is now described by the four equations (3.3), (3.4), (3.5), and (4.5). We may compare these equations with equations describing internal stellar structure.

The energy momentum tensor, describing matter at rest at each point, is

$$
\begin{aligned}
\theta_{\alpha \beta} & =\rho u_{\alpha} u_{\beta}+p\left(u_{\alpha} u_{\beta}-g_{\alpha \beta}\right), \\
u^{\alpha} & =\frac{d x^{\alpha}}{d s}=\left(u^{0}, 0,0,0\right) .
\end{aligned}
$$

Then [6] 


$$
\begin{gathered}
{\left[-\frac{\nu^{\prime \prime}}{2}+\frac{\nu^{\prime} \lambda^{\prime}}{4}-\frac{\nu^{\prime 2}}{4}-\frac{\nu^{\prime}}{r}\right]=K\left[\frac{\rho}{2}+\frac{3 p}{2}\right] e^{\lambda},} \\
{\left[\frac{\nu^{\prime \prime}}{2}-\frac{\nu^{\prime} \lambda^{\prime}}{4}+\frac{\nu^{\prime 2}}{4}-\frac{\lambda^{\prime}}{r}\right]=K\left[\frac{\rho}{2}-\frac{p}{2}\right] e^{\lambda},} \\
{\left[\frac{1}{r^{2}}+\frac{\nu^{\prime}-\lambda^{\prime}}{2 r}\right]-\frac{e^{\lambda}}{r^{2}}=K\left[\frac{\rho}{2}-\frac{p}{2}\right] e^{\lambda} .}
\end{gathered}
$$

There are two fields opposing gravitational contraction in both the soliton and the star, namely: $R(r)$ and $F\left[R^{2}\right]$ in the one case of $p(r)$ and $\rho(r)$ in the other case.

If the gravitational field is the same in the two cases, one may try to describe the fundamental fields $R(r)$ and $F\left[R^{2}\right]$ in terms of two effective or phenomenological fields, $p$ and $\rho$, by equating the right-hand sides of the two sets (3.3)-(3.5) and (5.2) $-(5.4)$. Then

$$
\begin{gathered}
F=\rho+3 p-\frac{\omega^{2}}{c^{2}} R^{2} e^{-\nu}, \\
-F e^{\lambda}+\left(R^{\prime}\right)^{2}=(\rho-p) e^{\lambda}, \\
F=p-\rho .
\end{gathered}
$$

The match described by Eqs. (5.5)-(5.7) shows that the soliton and stellar problems are really not comparable since Eqs. (5.6) and (5.7) imply

$$
R^{\prime}=0
$$

An additional difference between the two situations lies in the existence in the stellar case of a distinguished surface at which the internal and external solutions must match, while in the soliton case there is no corresponding surface. Since there is little to be learned, at least about the scalar case, from the stellar analogue, let us return to the soliton problem.

\section{BOUNDARY CONDITIONS}

At large distances we require the vanishing of $\psi(r)$ and $\psi^{\prime}(r)$ :

$$
\lim _{r \rightarrow \infty} R(r)=\lim _{r \rightarrow \infty} R^{\prime}(r)=0 .
$$

Then this condition and Eq. (3.6) imply

$$
\lim _{r \rightarrow \infty}\left(\nu^{\prime}+\lambda^{\prime}\right)=0
$$

or

$$
\lim _{r \rightarrow \infty}(\nu+\lambda)=\text { const. }
$$

By Eq. (3.7)

$$
\lim _{r \rightarrow \infty}\left[\frac{\lambda^{\prime}}{r}+\frac{e^{\lambda}-1}{r^{2}}-\frac{K}{2} F\left(R^{2}\right) e^{\lambda}\right]=0 .
$$

Then

$$
\lim _{r \rightarrow \infty}\left[\frac{\lambda^{\prime}}{r}+\frac{e^{\lambda}-1}{r^{2}}\right]=0
$$

if we assume

$$
\lim _{r \rightarrow \infty} F\left[R^{2}(r)\right]=0
$$

The preceding equation holds if we assume that $F\left[R^{2}\right]$ is a polynomial without a constant term, since we expect $R(r)$ to vanish exponentially. Equation (6.5) may be solved to give

$$
e^{-\lambda} \rightarrow 1-\frac{2 m}{r}
$$

and choosing the constant in Eq. (6.3) to vanish one has

$$
e^{\nu} \rightarrow 1-\frac{2 m}{r}
$$

Therefore at great distances the gravitational field becomes the Schwarzschild field, corresponding to

$$
\begin{aligned}
& \lambda \rightarrow \frac{2 m}{r}, \\
& \nu \rightarrow-\frac{2 m}{r} .
\end{aligned}
$$

Here $m$ is the geometric mass and is related to the usual mass $M$, by $m c^{2}=k M$, where $k$ is Newton's constant. Then the equation of motion (4.5) becomes at great distances

$$
R^{\prime \prime}+\frac{2}{r} R^{\prime}+\frac{\omega^{2}}{c^{2}} R+\frac{\partial F}{\partial R}=0
$$

since $\nu^{\prime}$ and $\lambda^{\prime}$, as well as $\nu$ and $\lambda$, all vanish at great distances.

Equation (6.11) agrees with the special relativistic equations as $r \rightarrow \infty$.

The key question is now whether the finiteness boundary condition can be satisfied at the origin.

By Eq. (3.6) $(\nu+\lambda)$ is an even function of $r$. Therefore set

$$
\begin{gathered}
\lambda=l_{0}+l_{2} r^{2}+l_{4} r^{4}+\cdots, \\
\nu=n_{0}+n_{2} r^{2}+n_{4} r^{4}+\cdots .
\end{gathered}
$$

Then by Eq. (3.6) to lowest order on the left

$$
2\left(l_{2}+n_{2}\right)+4\left(l_{4}+n_{4}\right) r^{2}=-\frac{K}{2}\left[\left(R^{\prime}\right)^{2}+\frac{\omega^{2}}{c^{2}} e^{\lambda-\nu} R^{2}\right]
$$

and

$$
4\left(l_{2}+n_{2}\right)=-K\left[R^{\prime}(0)^{2}+\frac{\omega^{2}}{c^{2}} R(0)^{2}\right] .
$$


We require that $R(r)$ be finite at $r=0$. We also require that $R^{\prime}(r)$ vanish at $r=0$; otherwise spherical symmetry would imply a cusp in $R(r)$ at $r=0$. Therefore

$$
R^{\prime}(0)=0 .
$$

To satisfy Eqs. (6.14) and (6.15) set

$$
l_{2}+\eta_{2}=-\frac{K}{4} \frac{\omega^{2}}{c^{2}} R(0)^{2} .
$$

Then also by Eq. (6.15)

$$
R(r)=a_{0}+a_{2} r^{2}+\cdots .
$$

By Eqs. (6.12) and (6.17), Eq. (3.7) now reads to lowest order

$$
\begin{aligned}
3 l_{2}+5 l_{4} r^{2}-\frac{K}{2}\left(F_{0}+F_{1} r+F_{2} r^{2}\right)\left(1+l_{0}+l_{2} r^{2}\right) \\
+K\left[a_{2}^{2} r^{2}+\frac{1}{4} \frac{\omega^{2}}{c^{2}}\left(a_{0}^{2}+2 a_{0} a_{2} r^{2}\right)\right]=0,
\end{aligned}
$$

where

$$
F\left[R^{2}(r)\right]=F_{0}+F_{1} r+F_{2} r^{2} .
$$

Hence

$$
\begin{gathered}
3 l_{2}-\frac{K}{2} F_{0}\left(1+l_{0}\right)+\frac{1}{4} K \frac{\omega^{2}}{c^{2}} a_{0}^{2}=0, \\
F_{1}=0, \\
5 l_{4}-\frac{K}{2}\left(F_{2}\left(1+l_{0}\right)+F_{0} l_{2}\right)+K a_{2}^{2}+\frac{K}{2} \frac{\omega^{2}}{c^{2}} a_{0} a_{2}=0 .
\end{gathered}
$$

At this point we must make some assumptions about the functional $F\left[R^{2}\right]$. Let

$$
F\left[R^{2}(r)\right]=A+\frac{B}{2} R^{2}+\frac{C}{4} R^{4},
$$

where

$$
A=0, \quad C>0, \quad B<0 .
$$

For comments on this mathematical rather than physical choice see Secs. X and XI. Then

$$
\begin{aligned}
F\left(a_{0}+a_{2} r^{2}\right)= & \frac{B}{2}\left(a_{0}^{2}+2 a_{0} a_{2} r^{2}\right) \\
& +\frac{C}{4}\left(a_{2}^{4}+4 a_{0}^{3} a_{2} r^{2}\right)+O\left(r^{4}\right)
\end{aligned}
$$

and

$$
\begin{aligned}
& F_{0}=\frac{B}{2} a_{0}^{2}+\frac{C}{4} a_{0}^{4}=F(R(0)), \\
& F_{2}=B a_{0} a_{2}+\frac{C}{2} a_{0}^{3} a_{2} .
\end{aligned}
$$

Equations (6.20) and (6.22) now determine $\lambda(r)$ in terms of $R(r)$ near $r=0 . \nu(r)$ may then be found from Eq. (3.6) in the same region. All of these functions appear well-behaved near $r=0$.

\section{COMPLETE SOLUTIONS}

The exact equations that must be satisfied are the gravitational equations of motion (3.3)-(3.5) and the separate equation of motion (4.5). These may be restated as

$$
\begin{gathered}
\frac{\lambda^{\prime}}{r}=\frac{1-e^{\lambda}}{r^{2}}+\frac{K}{2} F e^{\lambda}-\frac{K}{4}\left[\left(R^{\prime}\right)^{2}+\frac{\omega^{2}}{c^{2}} e^{\lambda-\nu} R^{2}\right], \\
\frac{\nu^{\prime}}{r}=\frac{e^{\lambda}-1}{r^{2}}-\frac{K}{2} F e^{\lambda}-\frac{K}{4}\left[\left(R^{\prime}\right)^{2}+\frac{\omega^{2}}{c^{2}} e^{\lambda-\nu} R^{2}\right], \\
R^{\prime \prime}+\frac{2}{r} R^{\prime}+\frac{1}{2}\left(\nu^{\prime}-\lambda^{\prime}\right) R^{\prime}+\frac{\omega^{2}}{c^{2}} e^{\lambda-\nu} R+e^{\lambda} \frac{\partial F}{\partial R}=0 .
\end{gathered}
$$

The boundary conditions at $r=0$ will be chosen as follows:

$$
\lambda=\lambda^{\prime}=\nu^{\prime}=R^{\prime}=0 .
$$

By Eqs. (3.8), (6.9), and (6.10)

$$
\lambda(0)+\nu(0)<0,
$$

and by Eq. (7.4)

$$
\nu(0)<(0)
$$

The three functions $\lambda, \nu$, and $R$ are then codetermined by Eqs. (7.1)-(7.3).

In order to gain some preliminary information about the complete solution, let us consider the special relativistic limit: namely,

$$
\lambda=\nu=0
$$

which corresponds to the vanishing of the energy momentum tensor of the scalar field or to setting $K=0$. In this limit the equation of motion (4.4) or (4.5) becomes

$$
R^{\prime \prime}+\frac{2}{r} R^{\prime}+\frac{\partial \widetilde{F}}{\partial R}=0
$$

where $\widetilde{F}$ differs from $F$ by replacing $B$ by $\widetilde{B}=\omega^{2} / c^{2}+B$. Let us now study the soliton solutions of Eq. (7.7) with the idea that they are closely related to the soliton solutions of Eq. (7.3). To do this we introduce the following functional $H\left(R, R^{\prime}\right)$ :

$$
H \equiv \frac{1}{2}\left(R^{\prime}\right)^{2}+\tilde{F}(R)
$$

By Eq. (7.7) one has

$$
\frac{d H}{d r}=-\frac{2}{r}\left(R^{\prime}\right)^{2}
$$




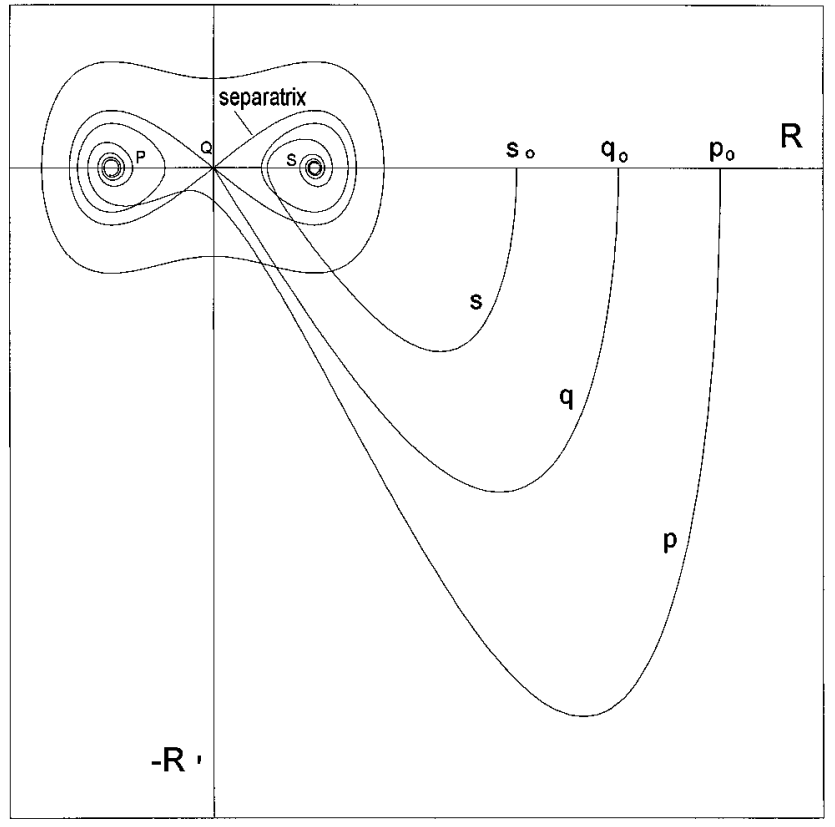

FIG. 1. Phase plane for the scalar field equation in the special relativistic limit.

The analysis of Eq. (7.7) depends on the portrait of $H\left(R, R^{\prime}\right)$ in the phase plane $\left(R, R^{\prime}\right.$ plane $)$ [3].

The closed curves in Fig. 1 are lines of constant $H$. If $H$ is represented as height above the phase plane, then the points $P$ and $S$ are minima and $Q$ is a saddle point, as we shall now show.

We have, by Eqs. (7.8) and (6.23),

$$
\begin{gathered}
\frac{\partial H}{\partial R}=\frac{\partial \widetilde{F}}{\partial R}=\tilde{B} R+C R^{3}, \\
\frac{\partial^{2} H}{\partial R^{2}}=\frac{\partial^{2} \tilde{F}}{\partial R^{2}}=\tilde{B}+3 C R^{2} .
\end{gathered}
$$

The extrema of $H$ in the $R$ direction are given by Eq. (7.10):

$$
\begin{gathered}
R=0, \quad R= \pm\left(-\frac{\tilde{B}}{C}\right)^{1 / 2} \\
\left(\frac{\partial^{2} H}{\partial R^{2}}\right)_{Q}=\tilde{B}<0 \\
\left(\frac{\partial^{2} H}{\partial R^{2}}\right)_{P, S}=-2 \widetilde{B}>0 .
\end{gathered}
$$

Therefore $Q$ is a maximum and $P, S$ are minima in the $R$ direction.

In the $R^{\prime}$ direction we have

$$
\frac{\partial H}{\partial R^{\prime}}=R^{\prime}=0 \quad \text { at } P, Q, \text { and } S,
$$

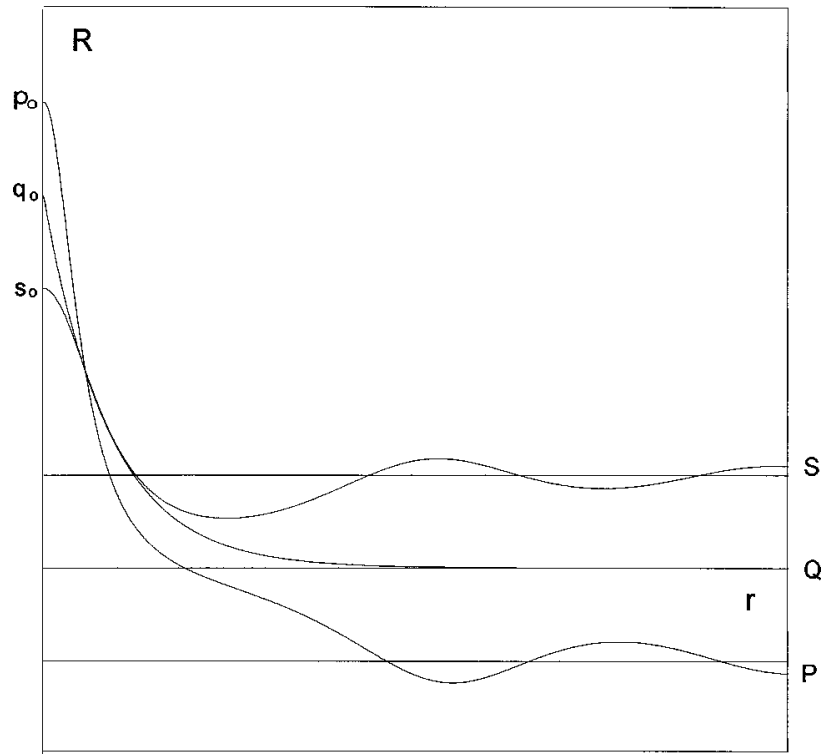

FIG. 2. The radial eigensolution $q$ and the bounding solutions $s$ and $p$, for the scalar field equation in the special relativistic limit. The points $P, Q$, and $S$ in Fig. 1 are represented by the straight lines $P, Q$, and $S$.

$$
\frac{\partial^{2} H}{\partial R^{\prime 2}}=1 \quad \text { at } P, Q, \text { and } S
$$

It follows that all three points $P, Q$, and $S$ are minima in the $R^{\prime}$ direction. Therefore $P$ and $S$ are minima in both directions and $Q$ is a saddle point. Combining this fact with Eq. (7.9), we see that a solution curve must always end at $P, Q$, or $S$ independent of its starting point. For example, the curves $s$ and $p$ spiral into the points $S$ and $P$.

This information in the phase plane may now be carried over to configuration space (Fig. 2). The points $S, P$, and $Q$ in Fig. 1 where $R^{\prime}=0$ represent the constant solutions of Eq. (7.7).

The curves $p, s$, and $q$ in Fig. 1 are now shown in Fig. 2 and

$$
\lim _{p_{0}-s_{0} \rightarrow 0}\left[\frac{R(p)+R(s)}{2}\right]=R(q) .
$$

As $p_{0}$ and $s_{0}$ are brought closer to $q_{0}$ the oscillatory behavior of $p$ and $s$ recedes to infinity and both $p$ and $s$ approach the eigensolution $q$, which terminates at the saddle point $Q$ with no oscillations. The eigensolutions are thus defined by the bounding classes of curves that terminate at $P$ and $S$ similar to the way that irrationals are defined by the Dedekind cut.

In this way the soliton solutions of Eq. (7.7) may be found. The same procedure may then be followed to obtain the corresponding solutions of Eq. (7.3). The soliton solutions of Eq. (7.6) and also of Eq. (7.3) are eigensolutions of a nonlinear differential equation. As such they differ fundamentally from the eigensolutions of a Schrödinger problem, since the normalization is not arbitrary but is itself an eigenvalue of the nonlinear equation. The soliton eigensolutions still determine the mass of the soliton just as the Schrödinger 


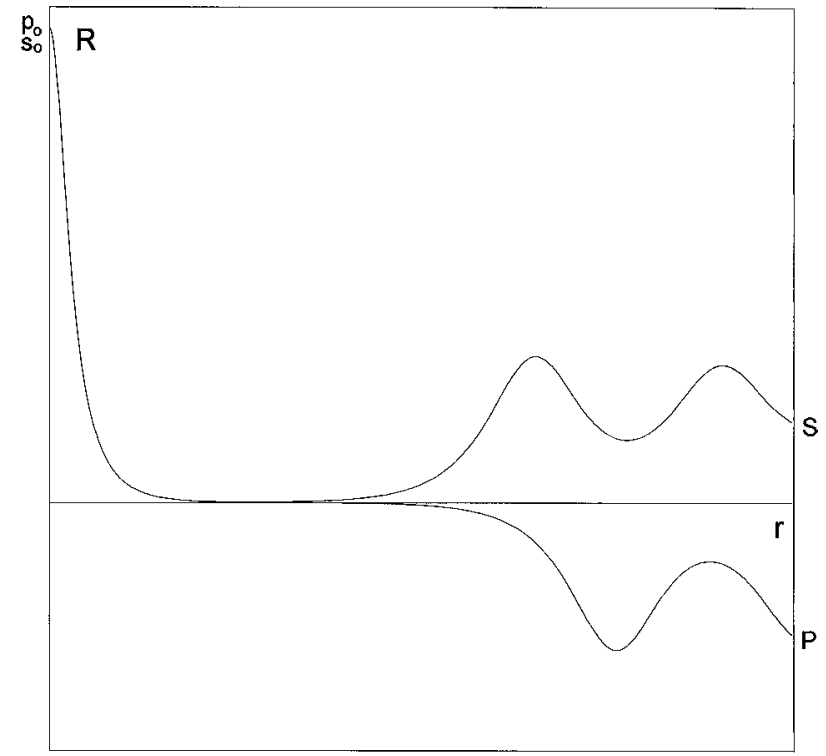

(a)

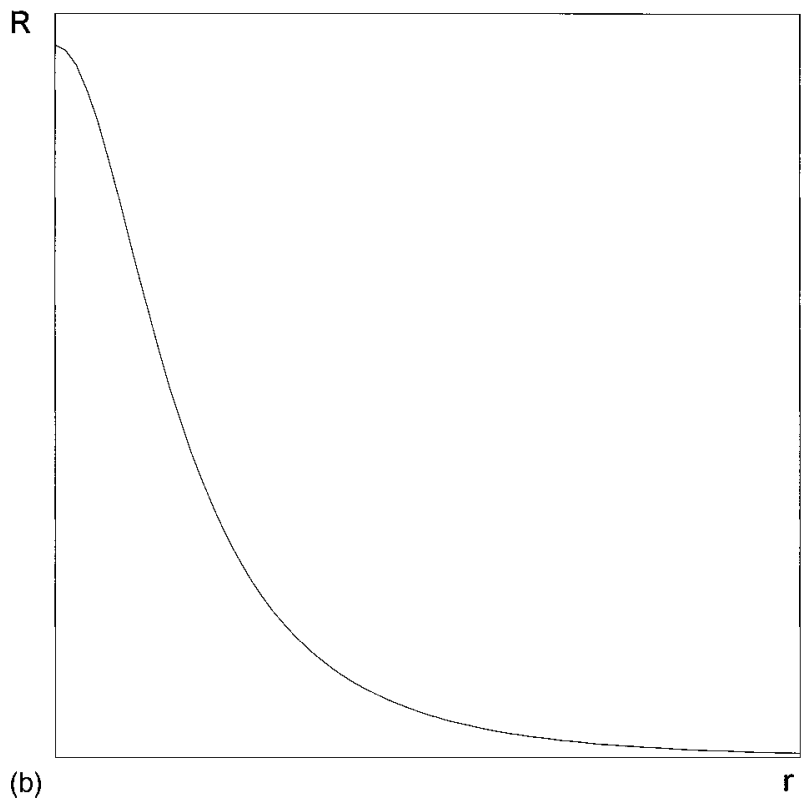

FIG. 3. (a) Numerical non-noding bounding solutions for the scalar equation coupled to gravity. The values of the parameters in Eq. (7.21) are $\delta=-0.0001$ (since $K$ is negative), $\beta^{\prime}=-0.001$, $\gamma^{\prime}=0.0005$, and $\hat{\omega}^{2}=0.0005$. (b) Eigensolution with assumed asymptotic form, corresponding to the bounding solutions in (a).

eigensolutions determine the energy of an atom. In general the soliton eigensolutions node in the same way as the Schrödinger wave functions.

We shall look for structures in which the solutions of Eq. (7.3) approximate the solutions of Eq. (7.7). Since these solutions must be determined numerically, let us next put Eqs. (7.1)-(7.3) in dimensionless form as follows:

$$
\begin{aligned}
\frac{d \lambda}{d x}= & \frac{1-e^{\lambda}}{x}-\frac{\delta}{4} x\left[\left(\frac{d y}{d x}\right)^{2}+\hat{\omega}^{2} e^{\lambda-\nu} y^{2}\right] \\
& +\frac{1}{2} x e^{\lambda}\left[\frac{\beta}{2} y^{2}+\frac{\gamma}{4} y^{4}\right],
\end{aligned}
$$

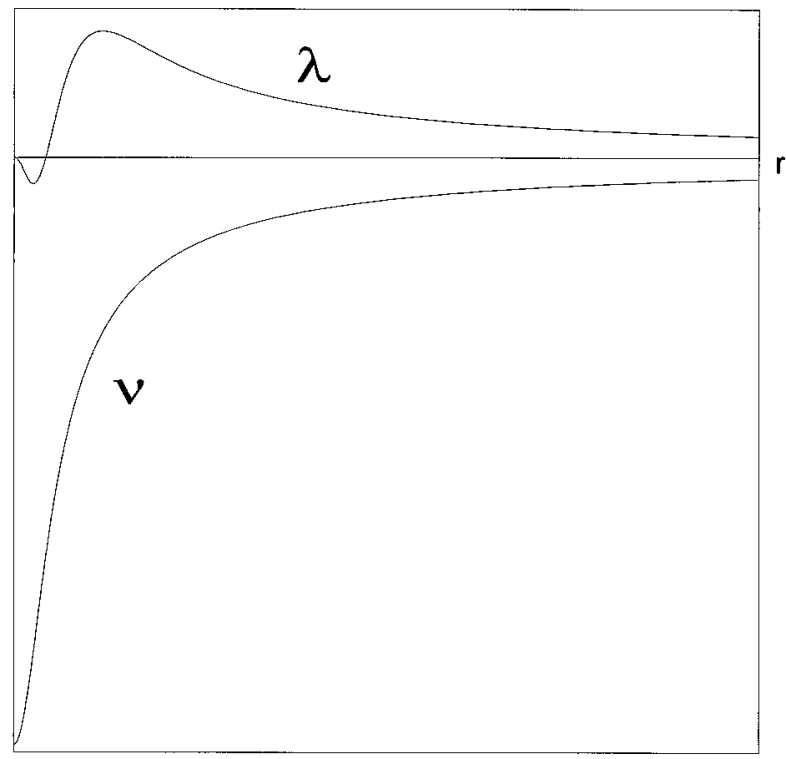

FIG. 4. $\lambda$ and $\nu$ corresponding to the eigensolution in Figure 3(b).

$$
\begin{aligned}
\frac{d \nu}{d x}= & \frac{e^{\lambda}-1}{x}-\frac{\delta}{4} x\left[\left(\frac{d y}{d x}\right)^{2}+\hat{\omega}^{2} e^{\lambda-\nu} y^{2}\right] \\
& -\frac{1}{2} x e^{\lambda}\left[\frac{\beta}{2} y^{2}+\frac{\gamma}{4} y^{4}\right], \\
\frac{d^{2} y}{d x^{2}}= & {\left[\frac{1-e^{\lambda}}{x}+\frac{1}{2}\left(\frac{\beta}{2} y^{2}+\frac{\gamma}{4} y^{4}\right)-\frac{2}{x}\right] \frac{d y}{d x} } \\
& -\left[\beta^{\prime} y+\gamma^{\prime} y^{3}\right] e^{\lambda}-e^{\lambda-\nu} \hat{\omega}^{2} y,
\end{aligned}
$$

where

$$
\begin{aligned}
& x=r / r_{0}, \quad \beta=\frac{r_{0}^{2} K}{c^{2}} \psi_{0}^{2} B, \\
& y=\psi / \psi_{0}, \quad \gamma=\frac{r_{0}^{2} K}{c^{2}} \psi_{0}^{2} C, \quad \delta=\frac{K}{c^{2}} \psi_{0}^{2}, \\
& \hat{\omega}=\omega r_{0} / c, \quad \beta^{\prime}=r_{0}^{2} B, \quad \gamma^{\prime}=r_{0}^{2} \psi_{0}^{2} C .
\end{aligned}
$$

Here $r_{0}$ is a fundamental length and $\psi_{0}$ is a fundamental real field strength. There is a large class of solutions lying in the parameter space $(\beta, \gamma, \delta, \hat{\omega})$.

\section{NUMERICAL SOLUTIONS}

We have obtained numerical solutions of the coupled equations (7.18)-(7.20) with boundary conditions (7.4),(7.5) and different initial values of $R(r)$. The parameters in Eq. (7.21) are chosen appropriately to maximize the sensitivity and convergence of the numerical algorithm. In particular we take $\hat{\omega}^{2}<\beta^{\prime}$, to obtain the bounding solutions.

We find solutions similar in behavior to the special relativistic case presented in Fig. 2. As the initial value of $R(r)$ is increased we make the transition from a solution that tends towards point $S$ to one that tends toward point $P$. These "bounding solutions" are shown in Fig. 3(a). By bringing the initial points $p_{0}$ and $s_{0}$ together, one obtains the initial 


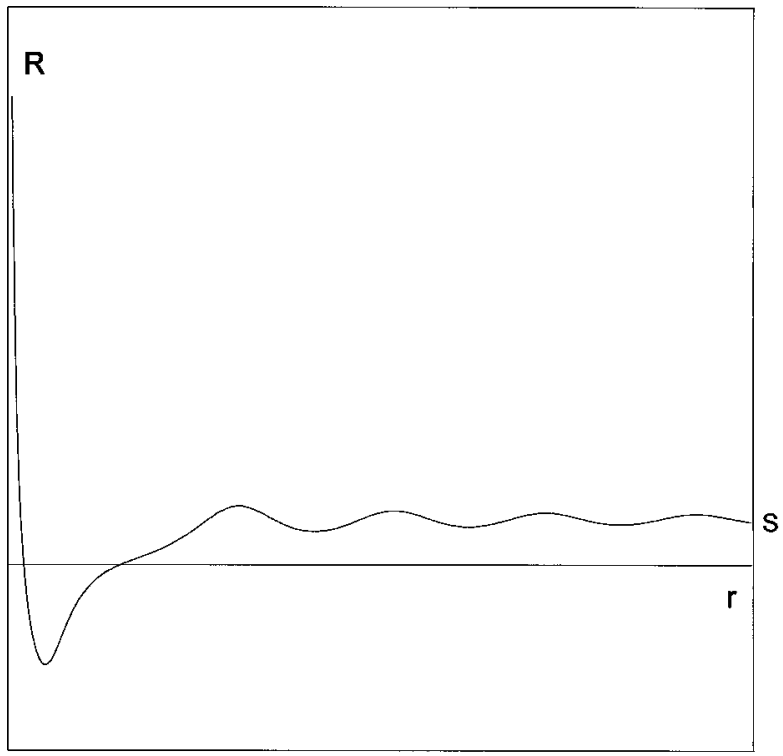

(a)

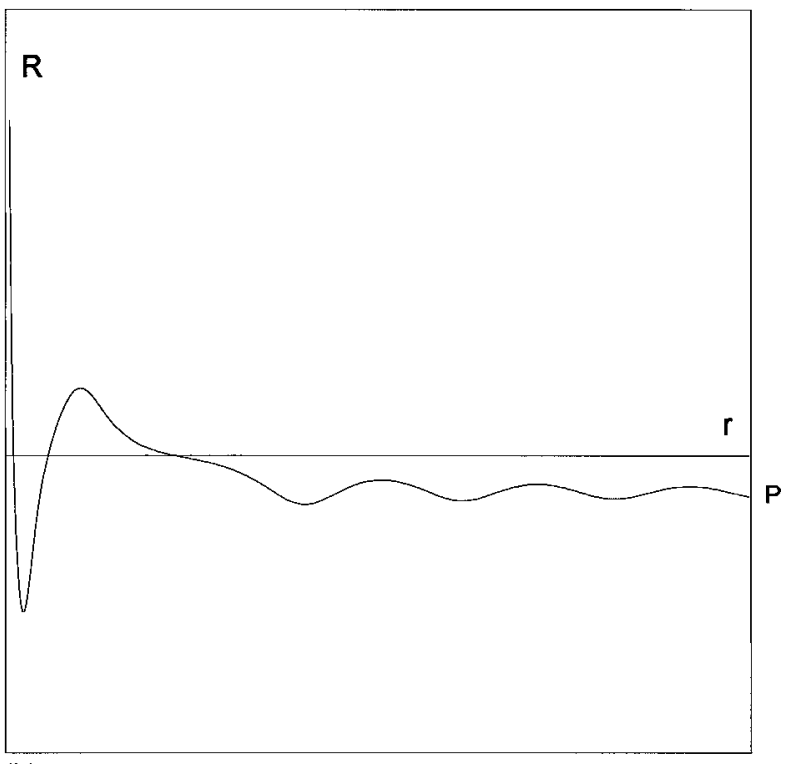

(b)

FIG. 5. (a) Numerical noding $(n=1)$ bounding solution which terminates at point $S$, for the scalar equation coupled to gravity. Same values for the parameters as in Fig. 3. (b) Numerical noding $(n=1)$ bounding solution which terminates at point $P$, for the scalar equation coupled to gravity. Same values for the parameters as in Fig. 3.

value of $R(r)$ belonging to an eigensolution. We obtain the eigensolution in Fig. 3(b) in the following manner. We define $r_{c}$ as the value of $r$ for which the two bounding solutions start to separate. For $r<r_{c}$ we take the average of the two bounding solutions as described in Eq. (7.17). For $r>r_{c}$ we assume the approximate asymptotic form $R \propto A e^{-a r} / r$. The parameters $a$ and $A$ are found by matching the values of $R$ and $R^{\prime}$ at $r=r_{c}$. To find the functions $\lambda(r)$ and $\nu(r)$ corresponding to the eigensolution, we use the asymptotic forms (6.9) and (6.10) as initial values for the integration which is performed backwards starting at $r_{\infty}=7198.5$, and feeding the values for the eigensolution previously obtained. The values for $m$ in the asymptotic forms (6.9) and (6.10) and for $r_{\infty}$ are constrained by requiring that the conditions at $r=0$ are satisfied. The results are presented in Fig. 4.

Continuing to increase the initial value of $R(r)$ for a fixed value of $\nu(r)$ we find noding bounding solutions that terminate at the two minima. Figure 5(a) shows a case that terminates at point $S$ while Fig. 5(b) shows the corresponding solution that terminates at point $P$. As in the nodeless case by bringing the initial points of the bounding solutions together we define a noding eigensolution. The procedure works as well here as for the special relativistic case.

\section{MASS}

At the special relativistic level of description the mass of a soliton is the integral of the energy density:

$$
M c^{2}=\int \theta_{00} d \vec{x}
$$

where $\theta_{00}$ is the energy density of a Lorentz invariant field.

When the source field is locked to the gravitational field in a composite structure, the contribution of the gravitational field to the mass must be taken into account. Since the energy and momentum of the gravitational field cannot be localized, however, there is no general relativistic tensor corresponding to the $\theta_{\mu \lambda}$, either for the gravitational field itself of for the source fields. Instead there is a so-called pseudoenergy-momentum tensor; and although this may be constructed in a variety of ways, it always leads, upon integration, to the same energy-momentum vector. For example, there is the original choice of Einstein:

$$
\Gamma=\sqrt{-g} g^{\sigma \rho}\left\{\Gamma_{\sigma \rho}^{\alpha} \Gamma_{\alpha \beta}^{\beta}-\Gamma_{\beta \rho}^{\alpha} \Gamma_{\alpha \sigma}^{\beta}\right\}
$$

in terms of which the total conserved energy-momentum vector of the soliton is

$$
P_{\mu}=-\frac{1}{K} \int \partial_{\lambda}\left[\frac{\partial \Gamma}{\partial\left(\partial_{\lambda} g^{\mu \nu}\right)} g^{0 \nu}\right] d \vec{x}
$$

integrated over all space. If the metric is independent of time, this three-dimensional integral may be converted to the surface integral

$$
P_{\mu}=-\frac{1}{K} \int_{S^{2}} \frac{\partial \Gamma}{\partial\left(\partial_{j} g^{\mu \nu}\right)} g^{0 \nu} \eta_{j} d S,
$$

where $S^{2}$ is the sphere at infinity.

In particular the energy is

$$
P_{0}=-\frac{1}{K} \int_{S^{2}} \frac{\partial \Gamma}{\partial\left(\partial_{j} g^{00}\right)} g^{00} \eta_{j} d S,
$$

since $g^{\mu \nu}$ is diagonal in our work.

The integrand may be simplified by the observation [6]

$\frac{\partial \Gamma}{\partial\left(\partial_{j} g^{00}\right)}=\frac{1}{2} \sqrt{-g}\left[g_{00} \partial_{\sigma} g^{\sigma j}-g_{00} g^{j \tau} g_{\sigma \rho} \partial_{\tau} g^{\sigma \rho}-g^{j \sigma} \partial_{\sigma} g_{00}\right]$.

To complete the evaluation of the energy integral one needs the form of the metric on the sphere at infinity. We have already seen that $\lambda$ and $\nu$, and therefore $g_{\mu \nu}$, acquire the 
Schwarzschild form at great distances. The Schwarzschild metric may in turn be written in the following isotropic form:

$$
d S^{2}=\left(\frac{1-\frac{m}{2 \rho}}{1+\frac{m}{2 \rho}}\right)^{2} d t^{2}-\left(1+\frac{m}{2 \rho}\right)^{4}\left(d x^{2}+d y^{2}+d z^{2}\right) .
$$

In terms of this metric, one has by Eq. (9.6)

$$
g^{00} \frac{\partial \Gamma}{\partial\left(\partial_{j} g^{00}\right)}=2 m \frac{x^{j}}{\rho^{3}}\left(1-\frac{m}{2 \rho}\right)
$$

and therefore by Eq. (9.5)

$$
P_{0}=m c^{2} / k=M .
$$

According to Eq. (6.9) $m$ is determined by the asymptotic shape of the curve $\lambda(r)$ in accordance with the equivalence principle.

\section{STABILITY AND MASS}

The Lagrangian of the nonlinear scalar field in flat space is

$$
\begin{aligned}
L & =\frac{1}{2} g^{\mu \lambda} \partial_{(\mu} \psi \partial_{\lambda)} \psi^{*}+F\left(|\psi|^{2}\right) \\
& \equiv T-V .
\end{aligned}
$$

The Hamiltonian density is

$$
\frac{1}{2} \Pi^{2}-F=\frac{1}{2} \Pi^{2}+V .
$$

Then $F=-V$ where $V$ is the usual potential. With the choice (6.24) the coefficient of $R^{4}$ in $V$ is negative so that $V$ is not positive definite and consequently $\psi$ is not physically acceptable at the quantum level as a free field. In our work, however, $\psi$ is not a free field; it is instead bound to the gravitational field and at the classical level it is only necessary that the complete structure representing the soliton have a positive mass. Strictly speaking the flat space limit is unphysical since it is not possible to turn off the universally coupled gravitational field. Alternatively the potential function $V$ may be made positive definite by adding a $R^{6}$ term with a positive coefficient. This would add two new saddle points outside the separatrix but would not essentially change the general argument, although it would limit the number of eigensolutions be restricting the initial point $R(0)$ to lie between the separatrix and the new saddle point.

Let us next compute the mass, which is now determined by Eqs. (9.9) and (6.7) rather than Eq. (9.1). The masses of the various noding solutions can therefore be read off from the $\lambda$ curves at large values of the radius. The three functions, $\lambda, \nu, R$, may be expanded in powers of $1 / r$ at large $r$ as follows:

$$
\lambda=\sum_{s=1}^{\infty} \frac{\hat{l}_{s}}{r^{s}}
$$

$$
\begin{aligned}
& \nu=\sum_{s=1}^{\infty} \frac{\hat{n}_{s}}{r^{s}}, \\
& R=\frac{e^{-\alpha r}}{r} \sum_{s=0}^{\infty} \frac{p_{s}}{r^{s}} .
\end{aligned}
$$

By Eqs. (6.3), (6.7), and (6.8), let us approximate Eqs. (10.4) and (10.5) by

$$
\begin{aligned}
& \lambda \cong \frac{2 m}{r}+\frac{a}{2 r^{2}}, \\
& \nu \cong \frac{-2 m}{r}-\frac{a}{2 r^{2}} .
\end{aligned}
$$

The solution of the linearized equation (7.3) in flat space is a spherical Hankel function:

$$
R(r)=\frac{e^{-\alpha r}}{r} .
$$

Let us carry $R$ to the same order in $r^{-1}$ as $\lambda$ and $\nu$. Then $R(r)$ becomes

$$
R(r)=\frac{e^{-\alpha r}}{r}\left(1+\frac{p_{1}}{r}\right),
$$

where $\alpha$ and $p_{1}$ are determined by the exact solutions of the nonlinear equation (7.3).

We may satisfy the linearized (7.3) to order $r^{-2}$ by substituting Eqs. (10.7), (10.8), and (10.9b) in this equation. We find

$$
\begin{aligned}
& \exp \left[\frac{4 m}{r}+\frac{a}{r^{2}}\right] \frac{\omega^{2}}{c^{2}} R+\frac{2 m}{r^{2}} R^{\prime}+\left(R^{\prime \prime}+\frac{2}{r} R^{\prime}\right) \\
& \quad+\exp \left[\frac{2 m}{r}+\frac{a}{2 r^{2}}\right] B R=0,
\end{aligned}
$$

where

$$
R^{\prime \prime}+\frac{2}{r} R^{\prime}=\left(\alpha^{2}+\frac{2 p_{1} \alpha}{r^{2}}\right) R
$$

by Eq. (10.9b) to terms of order $1 / r^{2}$. Then Eq. (10.10) implies the following relation:

$$
\begin{gathered}
{\left[\alpha^{2}+\frac{2 p_{1} \alpha}{r^{2}}\right]-\frac{2 m \alpha}{r^{2}}+\left[1+\frac{4 m}{r}+\frac{a+8 m^{2}}{r^{2}}\right] \frac{\omega^{2}}{c^{2}}} \\
+\left[1+\frac{2 m}{r}+\frac{a+4 m^{2}}{2 r^{2}}\right] B=0
\end{gathered}
$$

to the same order, or

$$
\alpha^{2}+\frac{\omega^{2}}{c^{2}}+B=0 \rightarrow B<0,
$$

$$
4 m \frac{\omega^{2}}{c^{2}}+2 m B=0 \rightarrow \frac{\omega^{2}}{c^{2}}=-\frac{1}{2} B \quad \text { and } \quad \alpha^{2}=\frac{\omega^{2}}{c^{2}},
$$




$$
2 p_{1} \alpha-2 m \alpha+a\left(\frac{\omega^{2}}{c^{2}}+\frac{B}{2}\right)+8 m^{2} \frac{\omega^{2}}{c^{2}}+2 m^{2} B=0 .
$$

By Eqs. (10.14) and (10.15)

$$
p_{1}-m+2 m^{2} \alpha=0
$$

and

$$
m=\frac{1}{4 \alpha}\left[1+\left(1-8 p_{1} \alpha\right)^{1 / 2}\right]
$$

If $p_{1}=0$,

$$
m=\frac{1}{2 \alpha}
$$

and

$$
R(r) \sim \frac{1}{r} e^{-r / m}
$$

Hence the mass, $m$, fixes the rate of decrease of both the gravitational field $\lambda(r)$ and the matter field $R(r)$ at large distances from the soliton. The value of $p_{1}$, as well as all the $p_{s}$, are determined by $R(0)$. Hence the mass of the soliton is fixed by the eigenvalue $R_{n}(0)$, where $n$ is the number of nodes.

Note also that these conclusions are compatible with the analysis of Sec. VII which requires that $\widetilde{B}=\omega^{2} / c^{2}+B$ be negative.

\section{DISCUSSION}

After completing this investigation we have learned of an extensive literature on boson stars [7]. Although the conceptual model employed in all of this literature is formally very similar to the model studied in our paper, the particular research closest to our work was carried out by Lee and collaborators [8] who have studied a class of general relativistic solitons generated by nonlinear scalar fields. They employ a different form of the self-interaction and more importantly a method of analysis completely different from that described in our paper. On the basis of their calculations they were led to discuss the possibility of cold, stable stellar configura- tions, called soliton stars. The present paper, on the other hand, was stimulated by the more recent string papers on solitonic black holes and our conjecture that there may be regular nontopological solitons in either these theories or in other fundamental completions of Einstein theory. As a preliminary to a more extended study we have based our work on a nonlinear field chosen for its simplicity rather than for its relation to a more fundamental theory.

The particular model studied in this paper was chosen in order to examine the influence of the gravitational field on the structure of a previously studied special relativistic soliton [3]. The present work does succeed in replicating the basic features of the special relativistic case (a singularityfree lump) with a discrete spectrum of eignesolutions. It also turns out that the method of analysis previously employed in the special relativistic case may be extended to the general relativistic problem.

The main contribution of the present paper probably turns on this last point, since the special method employed in the 1951 paper was restricted to a two-dimensional phase space while the current problem requires a six-dimensional phase space. Although it is still not clear how to construct a multidimensional dissipative function generalizing (7.8), we now know that exactly the same numerical procedure can be employed to determine the eigenfunctions and eigenvalues of the nonlinear scalar equation even when it is one member of a set of coupled differential equations. The extension of the numerical analysis in this way opens up the possibility of discussion more complex fields.

Finally we want to emphasize that the arguments of the present paper go through for nonlinear potentials with a lower bound, as remarked in the paragraph on stability.

Just as in a Schrödinger eigenvalue problem, we obtain the eigenfunctions by rejecting the irregular solutions. The resulting spectrum of eigenvalues (masses) is evidently very sensitive to the choice of the total field. Since the matter field in this paper was chosen for its simplicity and for easy comparison with the earlier study of the special relativistic case, and since it does not emerge from a fundamental theory, this model is by itself of mainly mathematical interest. On the other hand, our results as well as the results of Lee et al. and others makes it likely that nontopological solitons exist either in string models or in other fundamental completions of gravitational theory [9].
[1] A. Strominger, Nucl. Phys. B343, 167 (1990).

[2] M. Born and L. Infeld, Proc. R. Soc. London A144, 425 (1934).

[3] R. Finkelstein, R. Lelevier, and M. Ruderman, Phys. Rev. 83, 326 (1951).

[4] R. Friedberg, T. D. Lee, and A. Sirlin, Phys. Rev. D 13, 2739 (1976).

[5] G. 't Hooft, Nucl. Phys. B79, 276 (1974); A. M. Polyakov, JETP Lett. 20, 194 (1974).
[6] See, for example, R. Adler, M. Bazin, and M. Schiffer, Introduction to General Relativity (McGraw-Hill, New York, 1965).

[7] P. Jetzer, Phys. Rep. 220, 163 (1982); A. Liddle and M. Madsen, Int. J. Mod. Phys. D 1, 101 (1992).

[8] T. D. Lee, Phys. Rev. D 35, 3673 (1987); R. Friedberg, T. D. Lee, and Y. Pang, ibid. 35, 3640 (1987); 35, 3658 (1987).

[9] The solitons described in Ref. [1] are singularity free but topological. 